\section{. Junk DNA promotes sex chromosome evolution}

\section{S Matsunaga}

Heredity (2009) 102, 525-526; doi:10.1038/hdy.2009.36; published online 1 April 2009

S ex chromosomes evolved from a pair of autosomes, independently, in various phyla at different times. After the appearance of the gene involved in heterogametic male (XY) determination on the ancient $Y$ chromosome, extensive recombination suppression evolved between ancient sex chromosomes. Theoretically, the ancient $Y$ chromosome then suffered from a rapid accumulation of deleterious mutations and loss-of-function genes (Charlesworth et al., 2005). As a result, 'gene deserts' or gene-poor heterochromatin regions became distributed over the $\mathrm{Y}$ chromosome. As it is the fate of $\mathrm{Y}$ chromosomes to become inert and therefore, disappear from the male genome at some point in the future, they must be pitied.

Junk DNA, including transposable elements (TEs) and non-coding repetitive sequences, has been widely noted as an evolutionary force for producing novel gene function, and inducing chromosome rearrangements and genome diversification (Biemont and Vieira, 2006). Accumulation of these junk DNA sequences also contributes to the production of the gene deserts found in the Y chromosome. Kejnovskỳ et al. (2008) have recently discussed the potential for junk DNA accumulation to start at an early stage in the evolution of sex chromosomes. Both past cytogenetic analyses and recent genome projects have revealed that many animal $Y$ chromosomes have more abundant heterochromatin derived from repetitive sequences compared with $X$ chromosomes and autosomes. Accumulation of repetitive sequences induces abnormal recombination and chromosome breaks. Thus, junk DNA accumulation may well be a factor in the generation of differences in morphology and size observed between $\mathrm{X}$ and $\mathrm{Y}$ chromosomes; for example, in both the fruit fly Drosophila melanogaster and in humans, the $\mathrm{Y}$ chromosome is drastically smaller than the $X$ chromosome. The heterochromatic regions distributed over more than half of the human $\mathrm{Y}$ chromosome originated $\sim 300$ million years ago (m.y.a.), and the D. melanogaster Y chromosome, which was formed at least 60 m.y.a., has become almost entirely heterochromatic (Adams et al., 2000; Skaletsky et al., 2003). The Neo-Y chromosome of Drosophila miranda formed by a Y-autosome fusion only $1.2 \sim$ m.y.a. still harbors many functional genes. Even in this much younger $\mathrm{Y}$ chromosome, there is a more than 20-fold greater accumulation of repetitive sequences, mainly transposable elements, compared with that in the $\mathrm{X}$ chromosome (Bachtrog et al., 2008). These findings in animal species show that the accumulation of junk DNA is an important step in promoting the morphogenesis of sex chromosomes.

Junk DNA accumulation on Y chromosomes has been believed to be a symptom of Y-chromosome degeneration. Insertion of repetitive sequences into coding genes and regulatory regions induces alteration in the genes' functions and results in gene loss. However, there is no correlation between the insertion of the transposable element and gene dysfunction on the $\mathrm{Y}$ chromosome of D. miranda (Bachtrog et al., 2008). Moreover, the rate of gene gain on the Drosophila Y chromosomeusing the sequences of 12 species-is more than 10 times the rate of gene loss, in contrast with the mammalian $Y$ chromosome (Koerich et al., 2008). The contradiction between gene acquisition and accumulation of highly repetitive sequences on the Drosophila Y chromosomes, indicates that junk DNA accumulation is not always directly connected with $\mathrm{Y}$ chromosome degeneration.

To answer the question of how sex chromosomes are formed and evolve, we should survey more sex chromosomes in different taxa, including plants. The majority of plant species does not have sex chromosomes, making dioecious species (with male and female functions on separate plants) a minority, in contrast to animals. However, plant sex chromosomes have been found from moss to flowering plants, including familiar crop species, such as asparagus, hop, kiwi fruit, papaya and spinach
(Matsunaga and Kawano, 2001). The ancient $\mathrm{Y}$ chromosome in the liverwort Marchantia polymorpha is small and largely heterochromatic (Yamato et al., 2007), whereas most $Y$ chromosomes in flowering plants are the largest chromosomes in male genomes and many plant sex chromosomes are morphologically indistinguishable (Matsunaga, 2006; Jamilena et al., 2008). Why do sex chromosomes in flowering plants seem to retain their primitive characteristics similar to a pair of autosomes? One possible answer is that the plant sex chromosomes are evolutionarily very young. Thus, the study of sex chromosomes in flowering plants may allow us to catch a glimpse of the early stages of the genetic separation between males and females. For example, it will give us the opportunity to study the problem of whether junk DNA accumulation occurs before or after gene degeneration on $\mathrm{Y}$ chromosomes (Marais et al., 2008).

Kejnovskỳ et al. (2008) reported that the accumulation of repetitive sequences was generally found in the evolutionarily young plant Y chromosomes. The papaya $Y$ chromosome is the youngest in plants, having diverged from the $X$ chromosome only $2-3$ m.y.a. (Liu et al., 2004). Even in such young Y chromosomes, the accumulation of repetitive sequences and heterochromatinization can be detected.

The White Campion, Silene latifolia, is a flowering plant whose sex chromosomes were first discovered in 1923; the $\mathrm{Y}$ and $\mathrm{X}$ chromosomes are the largest and second-largest chromosomes of the male S. latifolia (Figure 1), respectively. The Y chromosome evolved in the Silene genus 10-20 m.y.a. through chromosomal inversion (Armstrong and Filatov, 2008). There is a large accumulation of microsatellites derived from repetitive sequences on the young and large $Y$ chromosomes, whereas transposable elements were found to be uniformly distributed along both sex chromosomes. Interestingly, chloroplast DNA also preferentially inserts into the $\mathrm{Y}$ chromosome. These studies strongly suggest that the accumulation of repetitive sequences is a crucial and common event in the early process of formation of plant and animal sex chromosomes, although the level of accumulation and types of repetitive sequences are varied. However, there is another possible plant-specific function of repetitive sequences during sex chromosome evolution because, unlike animals, plant genomes have increased through 


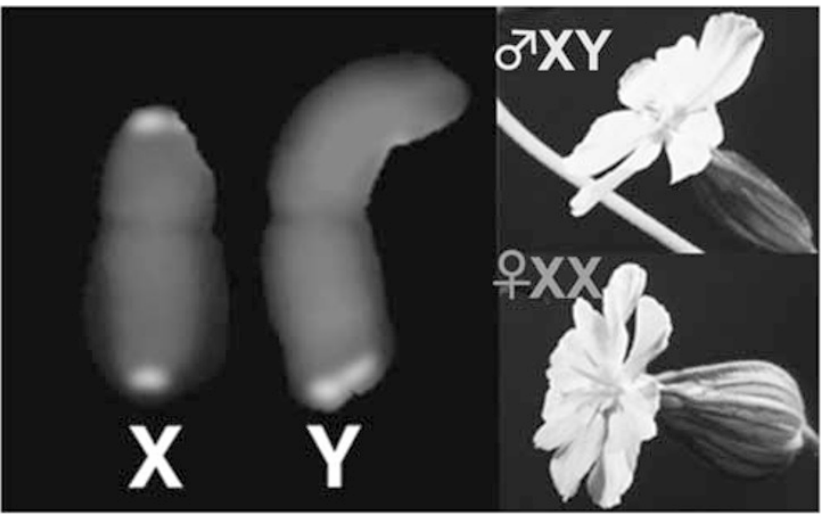

Figure 1 The large $\mathrm{X}$ and $\mathrm{Y}$ chromosomes in Silene latifolia. Yellow signals represent subtelomeric repetitive sequences. The right upper and lower flowers of S. latifolia are male and female with $\mathrm{XY}$ and $\mathrm{XX}$ chromosomes, respectively. The color reproduction of this figure is available on the html full text version of the manuscript.

repetition by whole genome duplication with amplification of repetitive sequences. Such junk DNA accumulation could contribute to increasing the size of plant Y chromosomes and keep the largest amount of DNA in the male genome. A more detailed investigation of young sex chromosomes in flowering plants promises new insights into fundamental issues of the birth and evolution of sex chromosomes.

Associate Professor S Matsunaga is at the Laboratory of Dynamic Cell Biology, Department of Biotechnology, Osaka University, Graduate School of Engineering, 2-1 Yamadaoka, Suita, Osaka, 565-8652, Japan.

e-mail: sachi@bio.eng.osaka-u.ac.jp
Adams MD, Celniker SE, Holt RA, Evans CA Gocayne JD, Amanatides PG et al. (2000). The genome sequence of Drosophila melanogaster. Science 287: 2185-2195.

Armstrong SJ, Filatov DA (2008). A cytogenetic view of sex chromosome evolution in plants. Cytogenet Genome Res 120: 241-246.

Bachtrog D, Hom E, Wong KM, Maside X, de Jong $P$ (2008). Genomic degradation of a young $Y$ chromosome in Drosophila miranda. Genome Biol 9: R30.

Biemont C, Vieira C (2006). Junk DNA as an evolutionary force. Nature 443: 521-524.

Charlesworth D, Charlesworth B, Marais G (2005) Steps in the evolution of heteromorphic sex chromosomes. Heredity 95: 118-128.

Jamilena M, Mariotti B, Manzano S (2008). Plant sex chromosomes: molecular structure and function. Cytogenet Genome Res 120: 255-264.

Kejnovskỳ E, Hobza R, Cermák T, Kubát Z, Vyskot B (2008). The role of repetitive DNA in structure and evolution of sex chromosomes in plants. Heredity 102: 533-541.

Koerich LB, Wang $X$, Clark AG, Carvalho AB (2008). Low conservation of gene content in the Drosophila Y chromosome. Nature 456: 949-951.

Marais GAB, Nicolas M, Bergero R, Chambrier P, Kejnovsky E, Moneger F et al. (2008). Evidence for degeneration of the $\mathrm{Y}$ chromosome in the dioecious plant Silene latifolia. Curr Biol 18: 1-5.

Matsunaga S, Kawano S (2001). Sex determination by sex chromosomes in dioecious plants. Plant Biol 3: 481-488.

Matsunaga S (2006). Sex chromosome-linked genes in plants. Genes Genet Syst 81: 219-226.

Liu Z, Moore PH, Ma H, Ackerman CM, Ragiba M, Yu Q et al. (2004). A primitive Y chromosome in papaya marks incipient sex chromosome evolution. Nature 427: 348-352.

Skaletsky H, Kuroda-Kawaguchi T, Minx PJ, Cordum HS, Hillier L, Brown LG et al. (2003). The male-specific region of the human $Y$ chromosome is a mosaic of discrete sequence classes. Nature 423: 825-837.

Yamato KT, Ishizaki K, Fujisawa M, Okada S, Nakayama S, Fujishita M et al. (2007). Gene organization of the liverwort $\mathrm{Y}$ chromosome reveals distinct sex chromosome evolution in a haploid system. Proc Natl Acad Sci USA 104: 6472-6477.

\section{Editor's suggested reading}

Greeff JM, Jansen van Vuuren GJ, Kryger P, Moore JC (2009). Outbreeding and possibly inbreeding depression in a pollinating fig wasp with a mixed mating system. Heredity 102: 349-356.

de Boer JG, Ode PJ, Vet LEM, Whitfield JB, Heimpel GE (2007). Diploid males sire triploid daughters and sons in the parasitoid wasp Cotesia vestalis. Heredity 99: 288-294.

Wilfert L, Gadau J, Schmid-Hempel P (2007). Variation in genomic recombination rates among animal taxa and the case of social insects. Heredity 98: 189-197. 